\title{
Differences between Lung Adenocarcinoma and Squamous Cell Carcinoma in Tumor Response and Survival after Induction Chemoradiotherapy followed by Surgery
}

Hiroaki Nomori ( $\nabla$ hnomori@qk9.so-net.ne.jp )

Kashiwa Kousei General Hospital

Atsushi Shiraishi

Kameda Medical Center

Koichi Honma

Kameda Medical Center

Kazufusa Shoji

Kameda Medical Center

Ayumu Otsuki

Kameda Medical Center

Yue Cong

Kameda Medical Center

Hiroshi Sugimura

Kameda Medical Center

Yu Oyama

Kameda Medical Center

Research article

Keywords: lung cancer, induction chemoradiotherapy, tumor response, survival, adenocarcinoma, squamous cell carcinoma

Posted Date: July 30th, 2020

DOI: https://doi.org/10.21203/rs.3.rs-47462/v1

License: (c) (1) This work is licensed under a Creative Commons Attribution 4.0 International License.

Read Full License 


\section{Abstract}

Objectives: To examine the differences in clinical response, pathological response, recurrence, and survival between lung adenocarcinoma (AD) and squamous cell carcinoma (SQ) after induction chemoradiotherapy (ICRT) followed by surgery.

Methods: Ninety-five patients with N1-2 or T3-4 non-small cell lung cancer were treated with ICRT followed by surgery between 2012 and 2018; 55 patients had AD and 40 had SQ. Differences in reductions of tumor size and fluorodeoxyglucose uptake on positron emission tomography after ICRT, as well as the pathological response, histological distribution of residual tumors, time to recurrence, and overall survival were compared between AD and SQ. Median follow-up period was 51 months.

Results: After ICRT, SQ showed significantly more reduction in both the tumor size and fluorodeoxyglucose uptake than $A D$ ( $p<0.001$ for both). Of the $40 \mathrm{SQ}, 22(55 \%)$ showed the pathological complete remission, which was significantly more frequent than 8 of 55 AD $(15 \%)(p<0.001)$. AD remained at the periphery of primary tumor in 39 of 55 patients $(71 \%)$, which was more frequently than 6 of 40 patients $(15 \%)$ in SQ $(p<0.001)$. While there was no significant difference in the rate of conducting postoperative adjuvant chemotherapy between $A D$ and SQ ( $47 \%$ vs. $40 \%$, respectively) $(p=0.48)$, the time to recurrence was significantly longer in SQ than in $A D(p=0.026 ; 3$-years recurrence-free rate: $77 \% \mathrm{vs.}$ $52 \%)$. However, overall survival was not significantly different between the two $(p=0.53 ; 3$-years survival rate: $74 \%$ in both).

Conclusion: While SQ showed better tumor response and longer time to recurrence after ICRT than $A D$, the overall survival was not different between the two. $A D$ remained at the periphery of primary tumor more frequently than SQ after ICRT.

\section{Background}

Induction chemoradiotherapy (ICRT) followed by surgery is a recently employed multimodal therapy for locally advanced non-small cell lung cancer (NSCLC), with satisfactory outcomes [1-5]. For survival prediction, the use of pathological response is better than the use of clinical response [6, 7]. However, only a limited number of studies have evaluated the differences in the tumor response and survival between adenocarcinoma (AD) and squamous cell carcinoma (SQ). Moreover, no studies have determined the differences in the location of residual tumors after ICRT between AD and SQ.

Therefore, the present study examined the following issues: (1) the differences in the clinical and pathological response after ICRT between AD and SQ; (2) the differences in time to recurrence and overall survival (OS) between the two; and (3) the difference in the histological distribution of residual tumors after ICRT between the two.

Methods 
The present study was a single-center retrospective observational study. The study design adhered to the Strengthening the Reporting of Observational Studies in Epidemiology guidelines [8]. Based on the Guidelines for Lung Cancer published by the Japanese Lung Cancer Society [9], we established a protocol for ICRT followed by surgery for patients with locally advanced NSCLC in October 2012, which was adopted by the institutional Lung Cancer Board. The retrospective analysis protocol for patients treated with ICRT followed by surgery was approved by the institutional ethics committee in 2014 (approval number: 14 -005). All patients provided a written informed consent after the attending physicians explained the risks and benefits of the ICRT followed by surgery. The written informed consent includes the consent to participate the study and publish the personal data without personal identification information, which includes clinical data, roentgen images, and pathological images.

\section{Eligibility}

The study participants for the present study fulfilled the following criteria: (1) N2 stage diagnosed via endobronchial ultrasound-guided transbronchial needle aspiration or fluorodeoxyglucose-positron emission tomography (FDG-PET); (2) N1 stage with metastatic lymph nodes invading the hilar bronchus or vessels, for the aim to avoid pneumonectomy; (3) T3 or T4 stage diagnosed via CT and magnetic resonance imaging, for the aim to decrease the local recurrence; (4) prediction of tolerance to ICRT followed by surgery; and (5) the patients preferred ICRT followed by surgery over definitive chemoradiotherapy. Tumor staging was based on the 8th edition of the TNM Classification proposed by the International Association for Study of Lung Cancer [10].

Protocol of induction chemoradiotherapy

A concurrent chemoradiotherapy, i.e. 2 cycles of a platinum doublet regimen and radiotherapy, was conducted. As a chemotherapy regimen, the combination with "cisplatin and docetaxel" was generally used for patients younger than 70 years old, and the combination with "carboplatin and paclitaxel" was for elderly patients. Radiation dose was usually $46 \mathrm{~Gy}$ for patients younger than 70 years old and $40 \mathrm{~Gy}$ for elderly patients.

Clinical response evaluation

The clinical response on CT was evaluated according to the Response Evaluation Criteria in Solid Tumors criteria [11]. The change in tumor size after ICRT was measured by "tumor size after ICRT/tumor size before ICRT".

Analysis of FDG-PET data

A PET/CT device (Discovery ST; GE Medical Systems, Amersham, UK) was used to perform FDG-PET scanning both before and after ICRT. The FDG uptake of the primary tumor was measured by using the 
standardized uptake value (SUV). The change in the SUV after ICRT was measured by "SUV after ICRT/SUV before ICRT".

Pathological response evaluation

Pathological findings on surgical materials were reviewed by a pathologist (K.H.), who had an experience of lung cancer pathology for more than 40 years. The pathological response was defined according to the criteria given in the "General Rules of Clinical and Pathological Records of Lung Cancer in Japan" as follows [12]: Ef.0 was defined as no therapeutic response; Ef.1a was defined as a viable tumor greater than or equal to two-thirds of the tumor; Ef.1b was defined as a viable tumor greater than or equal to onethird of the tumor and less than two-thirds; Ef.2 was defined as a viable tumor less than one-third of the tumor; and Ef.3 was defined as no viable tumor cells.

Measurement of the location of the external margin of residual tumors

Figure 1 shows a way of measurement of the location of the external margin of residual tumors. By examining the hematoxylin-eosin-stained sections of surgical materials, residual tumors were mapped on the grossly cut surface. Then, the location of the external margin of the residual tumor within the primary site was measured as follows: (1) the radius of the whole tumor ("a") and the distance between the center of the tumor and the external margin of the residual tumor ("b") were measured; and (2) the location of the external margin of the residual tumor was evaluated as "b/a".

Follow-up

Postoperative follow-up was conducted by body CT and brain magnetic resonance imaging every 3 or 4 months until 3 years after surgery and a minimum of every 6 months thereafter.

Data source

Between October 2012 and December 2018, a total of 107 patients with locally advanced NSCLC were planned to conduct ICRT followed by surgery. Follow-up data were collected from medical records in June 2020.

Study outcomes

The primary outcomes included the differences in the clinical tumor response, reduction in FDG-uptake, pathological tumor response, time to recurrence, and OS between AD and SQ. The secondary outcome was the difference in the location of the external margin of the residual tumor within the primary site between the two.

\section{Statistical analysis}

Differences in gender, age, comorbidity index, tumor size, tumor location (central vs. peripheral), SUV on PET, clinical stage, chemotherapy cycles, radiation doses, and surgical procedures between AD and SQ 
were examined by univariate and multivariate analyses. Differences of tumor size and SUV between preICRT and post-ICRT in each patient were analyzed by Wilcoxon singed rank test. Differences of numerical variables between the groups were analyzed by the Mann-Whitney U-test. Differences in nominal variables between the groups were analyzed using the $\chi^{2}$ test. Time to recurrence and OS after the initiation of ICRT were assessed via the Kaplan-Meier method [13]. All values in the text and tables are presented as means \pm standard deviation. P-values of $<0.05$ were considered statistically significant. All the analyses underwent on the SPSS software (IBM Ltd., New York, NY).

\section{Results}

Figure 2 shows the flowchart showing the patient selection process. Of the 107 patients treated with ICRT, surgery was not performed in 10 patients. Two patients were excluded because of tumor types other than AD or SQ. Finally, 95 patients were enrolled. The median duration from the completion of ICRT to surgery was 49 days (range: $20-67$ days). Of the 81 patients with $\mathrm{N} 1$ or 2 disease, the $\mathrm{N}$-stages were diagnosed via endobronchial ultrasound-guided needle biopsy in 48 patients (59\%) and via FDG-PET in 33 (41\%). Table 1 shows the characteristics of patients with $A D(n=55)$ and those with SQ $(n=40)$. With the multivariate analysis, SQ had larger tumor and localized in central bronchus more frequently than AD ( $P$ $=0.008$ and 0.005 , respectively), whereas there was no significant difference in gender, age, comorbidity index, clinical stage, number of chemotherapy cycles, radiation doses ( $40 \mathrm{~Gy}$ vs. $\geq 46 \mathrm{~Gy}$ ), and surgical procedures between the two $(p=0.90,0.13,0.17,0.35,0.91,0.72,0.60$, and 0.26 , respectively). The chemotherapy regimens were "carboplatin and paclitaxel" in 46 patients, "cisplatin and docetaxel" in 46, and "cisplatin and pemetrexed" in 3. Four AD patients and 2 SQ patients received just 1 cycle of chemotherapy, because of side effect. The radiation doses were $40 \mathrm{~Gy}$ for 49 patients, $46 \mathrm{~Gy}$ for $42,50 \mathrm{~Gy}$ for 2, and $60 \mathrm{~Gy}$ for 2 . The 2 patients who received $50 \mathrm{~Gy}$ had superior sulcus lung cancers. The 2 patients who received 60 Gy were treated by additional $14 \mathrm{~Gy}$ continuously after $46 \mathrm{~Gy}$, because of poor tumor shrinkage at the time.

Clinical TNM stages before ICRT did not show a significant difference of N0 stage between the groups, i.e. 11 of 55 patients $(20 \%)$ in $A D$ and 3 of $40(7.5 \%)$ in SQ $(p=0.14)$ (Table E1). There was no significant difference of T3/T4 stages between the groups, i.e. 32 of 55 patients (58.2\%) in AD and 30 of $40(75.0 \%)$ in SQ $(p=0.13)$.

The tumor size of both $A D$ and SQ significantly reduced after ICRT $(p<0.001)$, i.e. from $4.4 \pm 1.9 \mathrm{~cm}$ before ICRT to $3.2 \pm 1.6 \mathrm{~cm}$ after ICRT in AD, and from $5.7 \pm 1.9 \mathrm{~cm}$ before ICRT to $3.4 \pm 1.5 \mathrm{~cm}$ after ICRT in SQ (Fig. E1). Figure 3 shows the change of tumor size, which was $0.74 \pm 0.19$ ( $95 \%$ confidence interval [Cl]: 0.69-0.79; median value: 0.74$)$ in $A D$ and $0.61 \pm 0.18$ (95\% Cl: $0.54-0.64$; median value: 0.62 ) in SQ; the latter was significantly lower than the former $(p<0.001)$. In $A D, 25$ patients $(46 \%)$ showed a partial response and 30 (54\%) showed a stable disease; in SQ, 29 (73\%) showed a partial response and 11 (27\%) showed a stable disease (Table E2). The SQ had a higher partial response rate than $A D(p=0.016)$. 
The SUV of both AD and SQ significantly reduced after ICRT $(p<0.001)$, i.e. from $10.7 \pm 6.0$ before ICRT to $5.2 \pm 4.0$ after ICRT in AD, and from $14.5 \pm 6.1$ before ICRT to $4.2 \pm 3.5$ after ICRT in SQ (Fig. E2). Figure 4 shows the change ratio of SUV, which was $0.53 \pm 0.27$ (95\% Cl: $0.46-0.60$; median value: 0.48$)$ in $A D$ and $0.32 \pm 0.28$ (95\% Cl: $0.23-0.41$; median value: 0.23$)$ in SQ; the latter was significantly lower than the former $(p<0.001)$.

Lobectomy was performed in 88 patients, segmentectomy in 5, and pneumonectomy in 2 . For 6 patients with N3 stage, i.e. contralateral mediastinal nodes in 4 patients and supraclavicular nodes in 2, their N3 stations were dissected during surgery, all of which showed no metastasis. Of the 88 patients who underwent lobectomy, 18 required bronchial reconstruction. Fifty-one patients received the combined resection for T3/T4 disease. While 91 patients underwent complete resection (R0), the remaining 4 could not ( $\mathrm{R} 1$ resection in 2 and $\mathrm{R} 2$ in 2), because of tumor remnants in the aorta, right main bronchus, vertebra, and esophagus, respectively. While 93 patients were discharged without major complications, the remaining 2 patients died due to surgery-related complications.

The pathological tumor responses in AD were Ef.0-1 in 16 patients (29\%), Ef.2 in 31 (56\%), and Ef.3 in 8 (14\%), and in SQ, the responses were 3 (8\%), 15 (37\%), and 22 (55\%), respectively (Table E3). The SQ showed Ef.3 (complete remission) more frequently than AD $(p<0.001)$. The pathological N0 stage was seen in 33 of 55 AD cases (60.0\%) and 31 of 40 SQ cases (77.5\%), of which difference was not significant ( $p=0.07$ ) (Table E4). The down staging in N status was seen in 25 of 55 patients (45\%) in AD and 22 of 40 (55\%) in SQ, of which difference was not significant (Table E5, p = 0.35).

$A D$ showed residual tumors at the periphery of primary tumor more frequently than SQ as shown in Fig. 5, i.e. waterfall plots depicting the location of the external margin of residual tumors (b/a). Of the $55 \mathrm{AD}$ cases, $39(71 \%)$ showed the residual tumors at the periphery of primary tumor $(b / a=1.0)$ in contrast to only 6 of 40 SQ cases (15\%); the difference was significant $(p<0.001)$.

None of the patients were lost to follow-up. Median follow-up period was 51 months (range: 494 months). A total of 42 patients (44\%) received postoperative adjuvant chemotherapy; 26 of the 55 AD cases (47\%) and 16 of 40 SQ cases $(40 \%)$, of which difference was not significant $(p=0.48)$. During the study period, 40 patients experienced recurrences (AD in 31 and SQ in 9) and 35 patients died (AD in 19 and SQ in 16). Of the 40 patients with recurrences, local recurrence was observed in 3, i.e. 2 patients who underwent R2 resection and 1 who underwent R0. Among the 35 patients who died, 22 died of lung cancer and the other 13 died of other disease, including 2 who suffered surgery-related death. Figure 6 shows the time to recurrence, which was significantly longer in SQ than in $A D(p=0.026$, log-rank test), of which 3 -years recurrence-free rate was $77 \%$ and $52 \%$, respectively. For the patients with recurrence, additional chemotherapy was performed in 26 of the 31 AD cases (84\%) and 7 of the 9 SQ cases (78\%), of which difference was not significant $(p=0.62)$. Eventually, OS was not significantly different between $A D$ and $S Q$ as shown in Fig. 7 ( $p=0.53$, log-rank test), and the 3-years survival rate was $74 \%$ in both.

\section{Discussion}


The present study clarified the following points: (1) SQ showed better clinical and pathological responses after ICRT than AD; (2) time to recurrence was significantly longer in SQ than in $A D$, but there was no significant difference in OS between the two; and (3) AD showed the residual tumor at the periphery of primary tumor more frequently than SQ.

Only a few studies have evaluated the difference in clinical and pathological response between $A D$ and SQ after neoadjuvant treatments, including ICRT and chemotherapy alone. In the previous study by LiuJarin et al., among 30 patients, SQ cases showed better clinical and pathological responses than AD cases [14], similar to the results of our study. In contrast, Yamane et al. evaluated 53 patients and showed that there was no correlation between the histological type and pathological response [6]. The difference between the 2 reports was the frequency of using ICRT as neoadjuvant treatment, i.e. in the former study, ICRT was administered to 20 of the 30 patients (67\%), while in the latter study, ICRT was administered to only 13 of 53 patients (19\%), and chemotherapy alone for the remaining patients. This could be the reason why the data of the former study were similar to the results of our study, wherein ICRT was administered for $100 \%$ of the patients. Thus, we believe that SQ would be more sensitive to ICRT than AD.

While the time to recurrence was longer in $S Q$ than in $A D$, there was no significant difference in $O S$, regardless of the similar adjuvant chemotherapy and additional chemotherapy after recurrence, which could be caused by the following reasons: (1) AD would have less tumor-aggressiveness than SQ, resulting in longer survival after recurrence; and (2) AD might be more sensitive to chemotherapy, such as molecular targeted therapy, than SQ.

The pathological response after ICRT did not affect the OS in our study, which was similar to several previous reports. In a randomized study, Pless et al. treated 219 patients with N2-disease by using neoadjuvant chemotherapy alone vs. ICRT; despite a higher pathological response in the ICRT group, there was no significant difference in the 3-year survival rate between the two groups (45\% vs. $47 \%$, respectively) [15]. In another randomized study, Thomas et al. compared the survival of patients with stage III NSCLC treated with preoperative chemotherapy with and without additional ICRT; while the pathological response was higher in the patients treated with additional ICRT than in those without (60\% vs. $20 \%$ ), the 5 -year survival rate was not different (16\% vs. $14 \%$ ) [16]. Bharadwaj et al. retrospectively compared the outcomes of patients treated with standard dose and high-dose of radiotherapy in ICRT; although pathological complete remission was more frequent in the high-dose group, there was no significant difference in OS between the two groups [17]. Thus, the findings of the studies, including ours, suggest that the presence of systemic metastases affect the survival of patients with locally advanced NSCLC rather than the local control. However, intrathoracic recurrences after ICRT followed by surgery are reportedly few; intrathoracic recurrence was observed in only 5 of 51 patients (10\%) with chest wall invasion in the study by Kawaguchi et al. [4] and in 4 of 52 patients (8\%) with stage IIIA in the study by Bharadwaj et al. [17]. In the present study, intrathoracic recurrence was observed in only 3 of the 95 patients (3\%). Thus, we believe that while the radiation treatment in the induction therapy could not control the systemic recurrences, it could result in local control by extinguishing lymphatic permeation around the primary tumor and metastatic lymph nodes. 
No previous studies have evaluated the histological distribution of residual tumors after ICRT in NSCLC. The present study showed that the AD remained at the periphery of the primary tumor after ICRT more frequently than $S Q$, suggesting that the periphery of $A D$ is more resistant to ICRT than the periphery of SQ. The radio-sensitivity of tumors is reportedly dependent on intracellular oxygen concentration $[22,23]$. It is well known that the periphery of $A D$ frequently consists of well-differentiated tumor [24, 25], with less vascularity than SQ, which may therefore have a lower sensitivity to ICRT. Recently, intensity-modulated radiotherapy is being used to determine the planning of a radiation dose distribution (i.e., dose painting) within the primary tumor to prevent local recurrence of various tumors including NSCLC [26, 27]. The present study showed that AD frequently remained at the periphery of the primary tumor, while SQ did not; this finding could help to decide the dose distribution in intensity-modulated radiotherapy for NSCLC.

The present study had the following limitations: (1) although all patients received ICRT before surgery, the chemotherapy regimens and the radiation dose were not standardized; and (2) there was a heterogeneity in tumor size and TNM stages between AD and SQ. Because the present study lacked external validity, it should be assessed with future multicenter studies for the difference of tumor response and prognosis between $A D$ and $S Q$.

\section{Conclusion}

The present study showed a better tumor response and better recurrence-free survival in patients with SQ than in those with AD, but overall survival was not different. Although ICRT resulted in local control of locally advanced NSCLC, systemic chemotherapy might be more important than preoperative radiation therapy to improve the survival.

$>$

\section{Abbreviations}

$A D=$ adenocarcinoma

$\mathrm{CT}=$ computed tomography

ICRT = induction chemoradiotherapy

FDG = fluorodeoxyglucose

NSCLC $=$ non-small cell lung cancer

OS = overall survival

PET = positron emission tomography

RFS = recurrence-free survival 
$\mathrm{SQ}=$ squamous cell carcinoma

SUV = standardized uptake value

\section{Declarations}

- Ethics Approval and consent to participate:

The name of the study: Induction chemoradiotherapy for locally advanced non-small cell lung cancer.

Date and number of IRB approval: January $25^{\text {th }}$ in $2014,14-005$

- A written informed consent includes the patent's consent to participate the study, publish the personal data including clinical data, roentgen images, and pathological images.

- Data Availability Statement: Our data is available to be accessed.

- Author contributions: All authors contribute to at least one of study design, patient treatments, data collection, and statistics.

- Conflict of interest: The authors have no conflicts of interest or financial ties.

- Funding: No funding for the present study.

\section{References}

1. Albain KS, Swann RS, Rusch VW, Turrisi AT 3rd, Shepherd FA, Smith C,et al. Radiotherapy plus chemotherapy with or without surgical resection for stage III non-small-cell lung cancer: a phase III randomized controlled trial. Lancet. 2009;374:379-86.

2. Rusch VW, Giroux DJ, Kraut MJ, Crowley J, Hazuka M, Winton T, et al. Induction chemoradiation and surgical resection for superior sulcus non-small-cell lung carcinomas: long-term results of Southwest Oncology Group Trial 9416 (Intergroup Trial 0160). J Clin Oncol. 2007;25:313-8.

3. Kunitoh H, Kato H, Tsuboi M, Shibata T, Asamura H, Ichinose $Y$, et al. Japan Clinical Oncology Group. Phase II trial of preoperative chemoradiotherapy followed by surgical resection in patients with superior sulcus non-small-cell lung cancers: report of Japan Clinical Oncology Group trial 9806. J Clin Oncol. 2008;26:644-9.

4. Kawaguchi K, Yokoi K, Niwa H, Ohde Y, Mori S, Okumura S, Shiono S, Ito H, Yano M, Shigemitsu K, Hiramatsu Y, Okami J, Saito H. Trimodality therapy for lung cancer with chest wall invasion: initial results of a phase II study. Ann Thorac Surg. 2014;98:1184-91.

5. Eberhardt WE, Pöttgen C, Gauler TC, Friedel G, Veit S, Heinrich V, et al. Phase III study of surgery versus definitive concurrent chemoradiotherapy boost in patients with resectable stage IIIA(N2) and selected IIIB non-small-cell lung cancer after induction chemotherapy and concurrent chemoradiotherapy (ESPATUE). J Clin Oncol. 2015;33:4194-201. 
6. Yamane Y, Ishii G, Goto K, Kojima M, Nakao M, Shimada Y, et al. A novel histopathological evaluation method predicting the outcome of non-small cell lung cancer treated by neoadjuvant therapy: the prognostic importance of the area of residual tumor. J Thorac Oncol. 2010;5:49-55.

7. Junker K, Thomas M, Schulmann K, Klinke F, Bosse U, Müller KM. Tumor regression in non-small-cell lung cancer following neoadjuvant therapy. Histological assessment. J Cancer Res Clin Oncol. 1997;123:469-77.

8. Vandenvbroucke JP. von Elm E, Altman DG, Gøtzsche PC, Mulrow CD, Pocock SJ, Pool C, Schlesselman J, Egger M. Strengthening the Reporting of Observational Studies in Epidemiology (STROBE): Explanation and Elaboration. Epidemiology 2007; 18:805-35.

9. Guidelines for diagnosis and treatment of lung cancer. The Japan Lung Cancer Society. Tokyo: Kanehara Inc.; 2005.

10. Chansky K, Detterbeck FC, Nicholson AG, Rusch VW, Vallières E, Groome P, et al. IASLC Staging and Prognostic Factors Committee, Advisory Boards, and Participating Institutions. The IASLC Lung Cancer Staging Project: External Validation of the Revision of the TNM Stage Groupings in the Eighth Edition of the TNM Classification of Lung Cancer. J Thorac Oncol. 2017;12:1109-21.

11. Eisenhauer EA, Therasse P, Bogaerts J, Schwartz LH, Sargent D, Ford R, et al. New response evaluation criteria in solid tumors: revised RECIST guideline. Eur J Cancer. 2009;45:228-47.

12. General rule for clinical and pathological record of lung cancer. 7th ed. Tokyo: The Japan Lung Cancer Society, Kanehara, 2010.

13. Kaplan EL, Meier P. Non-parametric estimation from incomplete observations. J Am Stat Assoc. 1958;53:457-84.

14. Liu-Jarin X, Stoopler MB, Raftopoulos H, Ginsburg M, Gorenstein L, Borczuk AC. Histologic assessment of non-small cell lung carcinoma after neoadjuvant therapy. Mod Pathol. 2003 Nov;16(11):1102-8.

15. Früh M, Betticher DC, Stupp R, Xyrafas A, Peters S, Ris HB, et al, Swiss Group for Clinical Cancer Research (SAKK). Multimodal treatment in operable stage III NSCLC: A pooled analysis on long-term results of three SAKK trials (SAKK 16/96, 16/00, and 16/01). J Thorac Oncol. 2019;14:115-23.

16. Thomas M, Rübe $C$, Hoffknecht P, Macha HN, Freitag L, Linder A,.et al. German Lung Cancer Cooperative Group. Effect of preoperative chemoradiation in addition to preoperative chemotherapy: a randomized trial in stage III non-small-cell lung cancer. Lancet Oncol. 2008;9:636-48.

17. Bharadwaj SC, Vallières E, Wilshire CL, Blitz M, Page B, Aye RW. et al. Higher versus standard preoperative radiation in the trimodality treatment of stage Illa lung cancer. Ann Thorac Surg. 2015;100:207 - 13.

18. Shimosato Y, Hashimoto T, Kodama T, Kameya T, Suzuki A, Nishizaki Y, et al. Prognostic implications of fibrotic focus (scar) in small peripheral lung cancers. Am J Surg Pathol. 1980;4:365-73.

19. Noguchi M, Morikawa A, Kawasaki M, Matsuno Y, Yamada T, Hirohashi S, et al. Small adenocarcinoma of the lung. Histologic characteristics and prognosis. Cancer. 1995;75:2844-52. 
20. Jordan BF, Sonveaux P, Feron O, Grégoire V, Beghein N, Dessy C, et al. Nitric oxide as a radiosensitizer: evidence for an intrinsic role in addition to its effect on oxygen delivery and consumption. Int $\mathrm{J}$ Cancer. 2004;109:768-73.

21. Saleem W, Suzuki Y, Mobaraki A, Yoshida Y, Noda S, Saitoh Jl. set al. Reduction of nitric oxide level enhances the radiosensitivity of hypoxic non-small cell lung cancer. Cancer Sci. 2011;102:2150-6.

22. Jordan BF, Sonveaux P, Feron O, Grégoire V, Beghein N, Dessy C, et al. Nitric oxide as a radiosensitizer: evidence for an intrinsic role in addition to its effect on oxygen delivery and consumption. Int $\mathrm{J}$ Cancer. 2004;109:768-73.

23. Saleem W, Suzuki Y, Mobaraki A, Yoshida Y, Noda S, Saitoh Jl. set al. Reduction of nitric oxide level enhances the radiosensitivity of hypoxic non-small cell lung cancer. Cancer Sci. 2011;102:2150-6.

24. Shimosato $Y$, Hashimoto T, Kodama T, Kameya T, Suzuki A, Nishizaki Y, et al. Prognostic implications of fibrotic focus (scar) in small peripheral lung cancers. Am J Surg Pathol. 1980;4:365-73.

25. Noguchi M, Morikawa A, Kawasaki M, Matsuno Y, Yamada T, Hirohashi S, et al. Small adenocarcinoma of the lung. Histologic characteristics and prognosis. Cancer. 1995;75:2844-52.

26. Sachpekidis C, Thieke C, Askoxylakis V, Nicolay NH, Huber PE, Thomas M, et al. Combined use of (18)F-FDG and (18)F-FMISO in unresectable non-small cell lung cancer patients planned for radiotherapy: a dynamic PET/CT study. Am J Nucl Med Mol Imaging. 2015;5:127-42.

27. Askoxylakis V, Dinkel J, Eichinger M, Stieltjes B, Sommer G, Strauss LG, Dimitrakopoulou-Strauss A, Kopp-Schneider A, Haberkorn U, Huber PE, Bischof M, Debus J, Thieke C. Multimodal hypoxia imaging and intensity modulated radiation therapy for unresectable non-small-cell lung cancer: the HIL trial. Radiat Oncol. 2012;7:157.

\section{Figures}




\section{Measurement of the external side of residual viable tumor (b/a)}

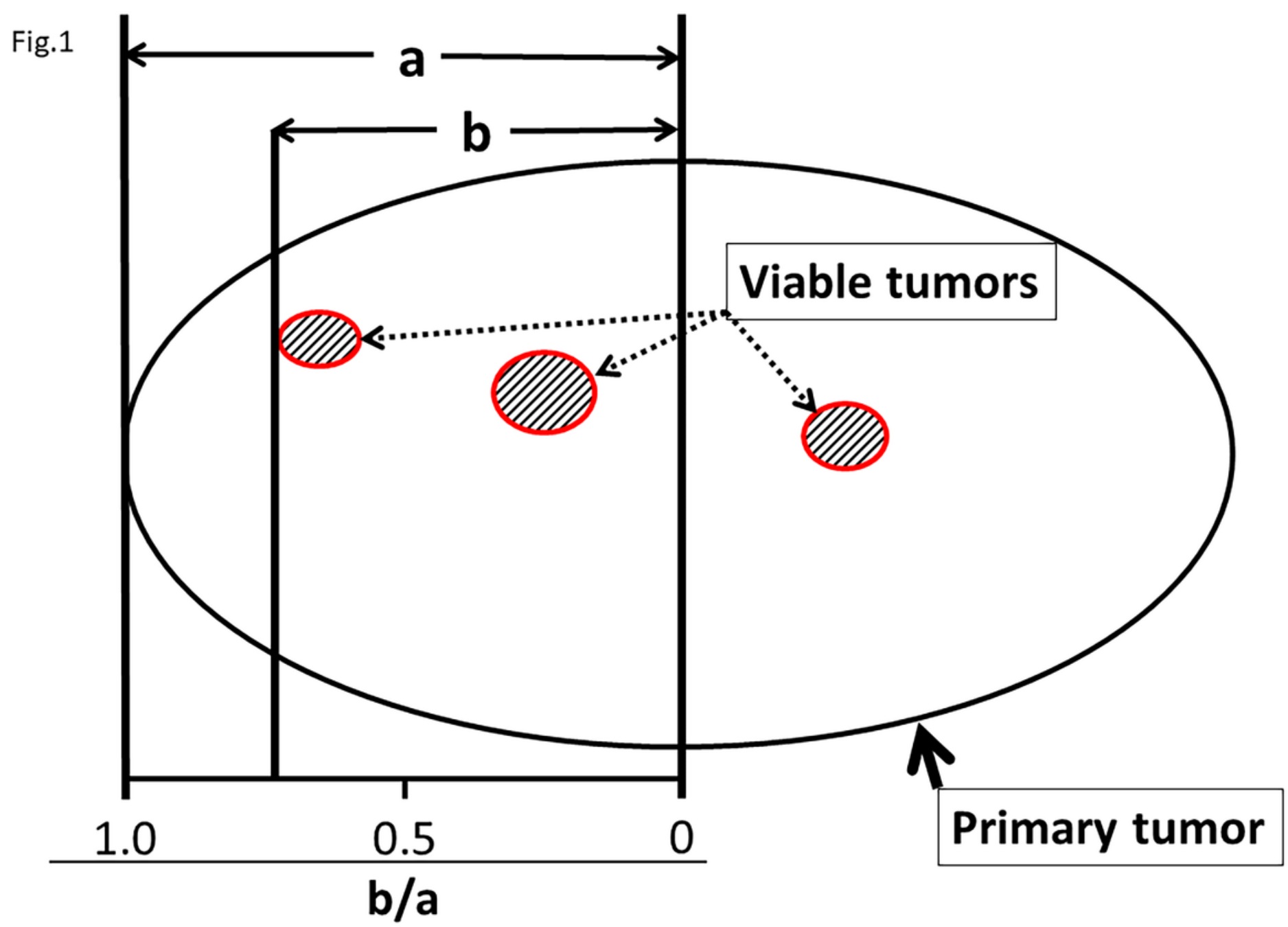

Figure 1

Measurement of the external margin of the residual tumor. A "a" is the radius of the whole tumor and " $b$ " is the distance between the center of the whole tumor and the external margin of the residual tumor. External margin of the residual tumor $=b / a$ ). 


\section{Flowchart}

Fig.2

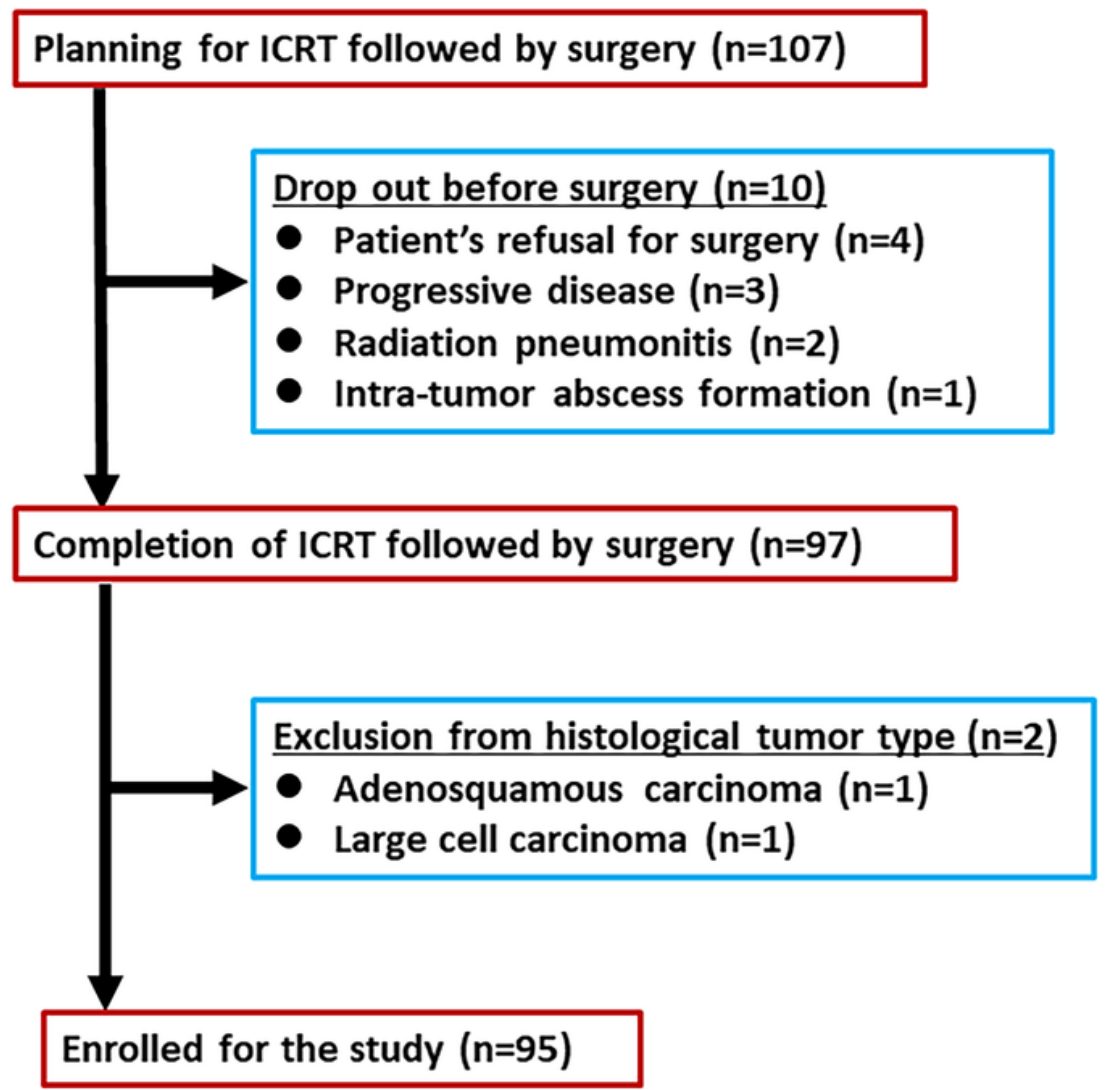

Figure 2

Flowchart showing the patient selection process. 
Fig.3

\section{Change in tumor size after ICRT}

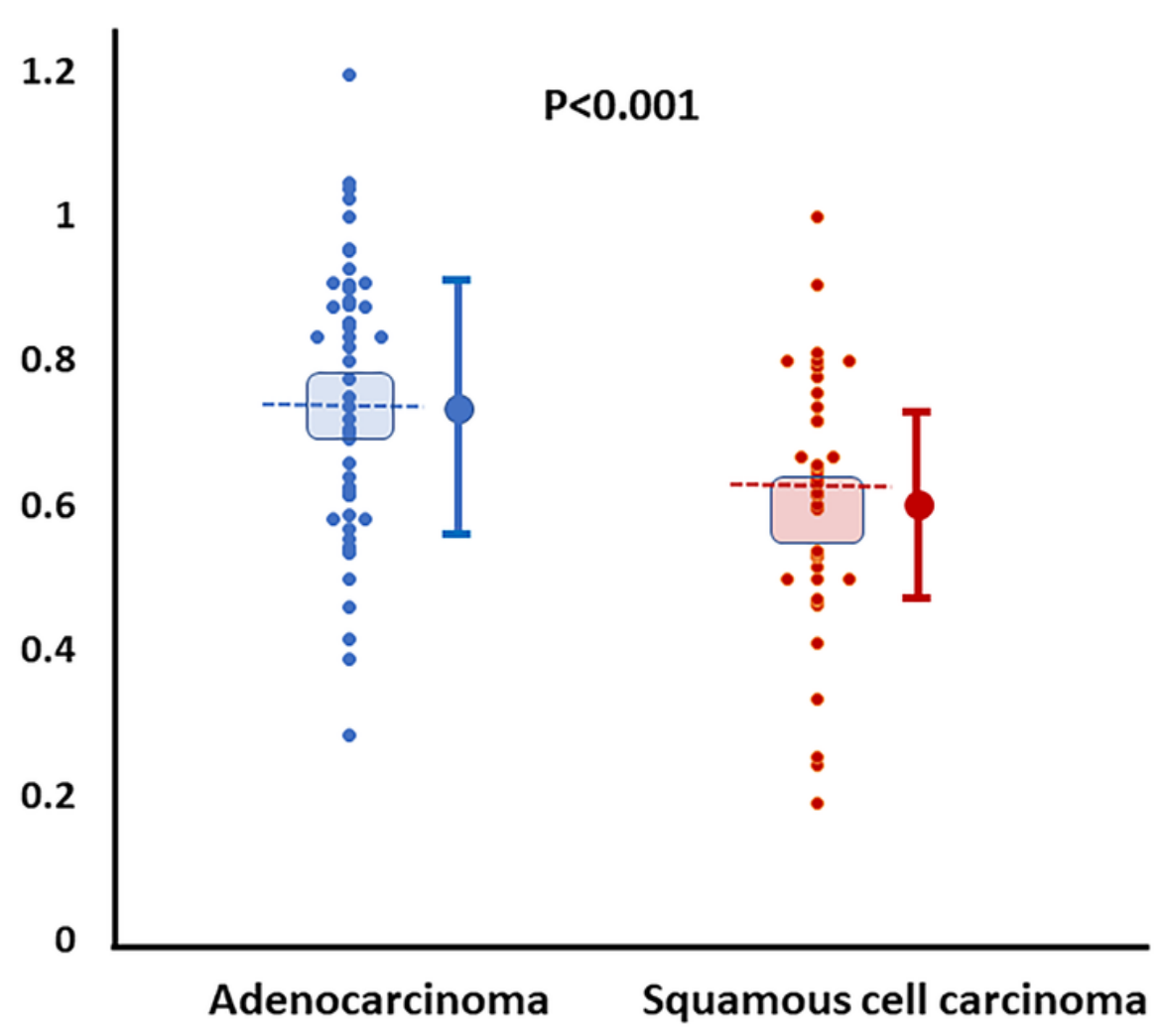

Figure 3

Changes in tumor size after induction chemoradiotherapy for adenocarcinoma and squamous cell carcinoma. Squamous cell carcinoma showed significantly more reduction in tumor size than adenocarcinoma $(p<0.001)$. The $95 \%$ confidence intervals in each size change are shown as shade in each color. The dotted line shows the median value. 
Fig.4

Change in SUV after ICRT

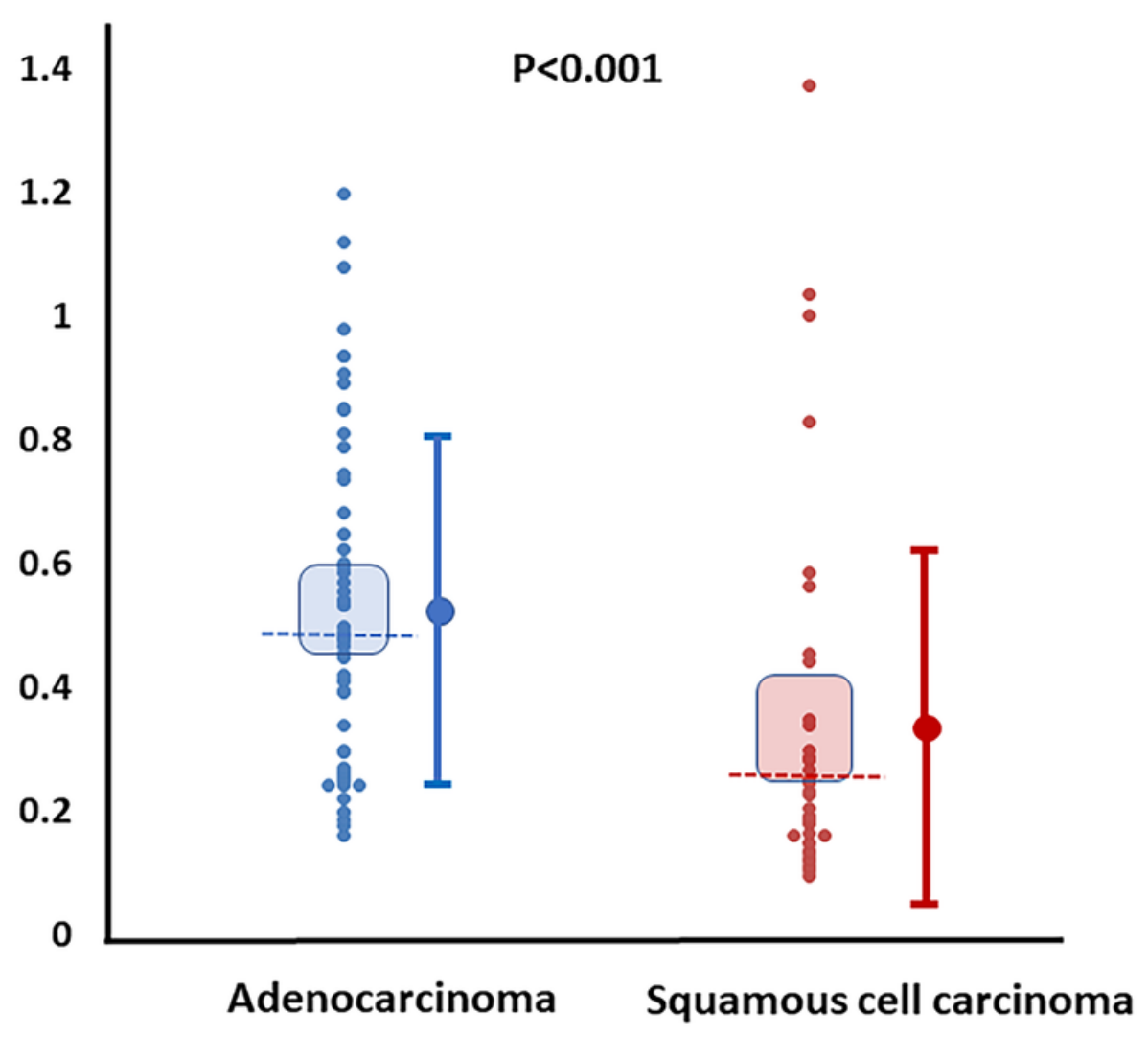

Figure 4

Changes in standard uptake value after induction chemoradiotherapy for adenocarcinoma and squamous cell carcinoma. Squamous cell carcinoma showed significantly more reduction in standard uptake value than adenocarcinoma $(p<0.001)$. The $95 \%$ confidence intervals in each SUV change are shown as shade in each color. The dotted line shows the median value. 
Fig.5

\section{Location of the external margin of residual tumors in primary site}
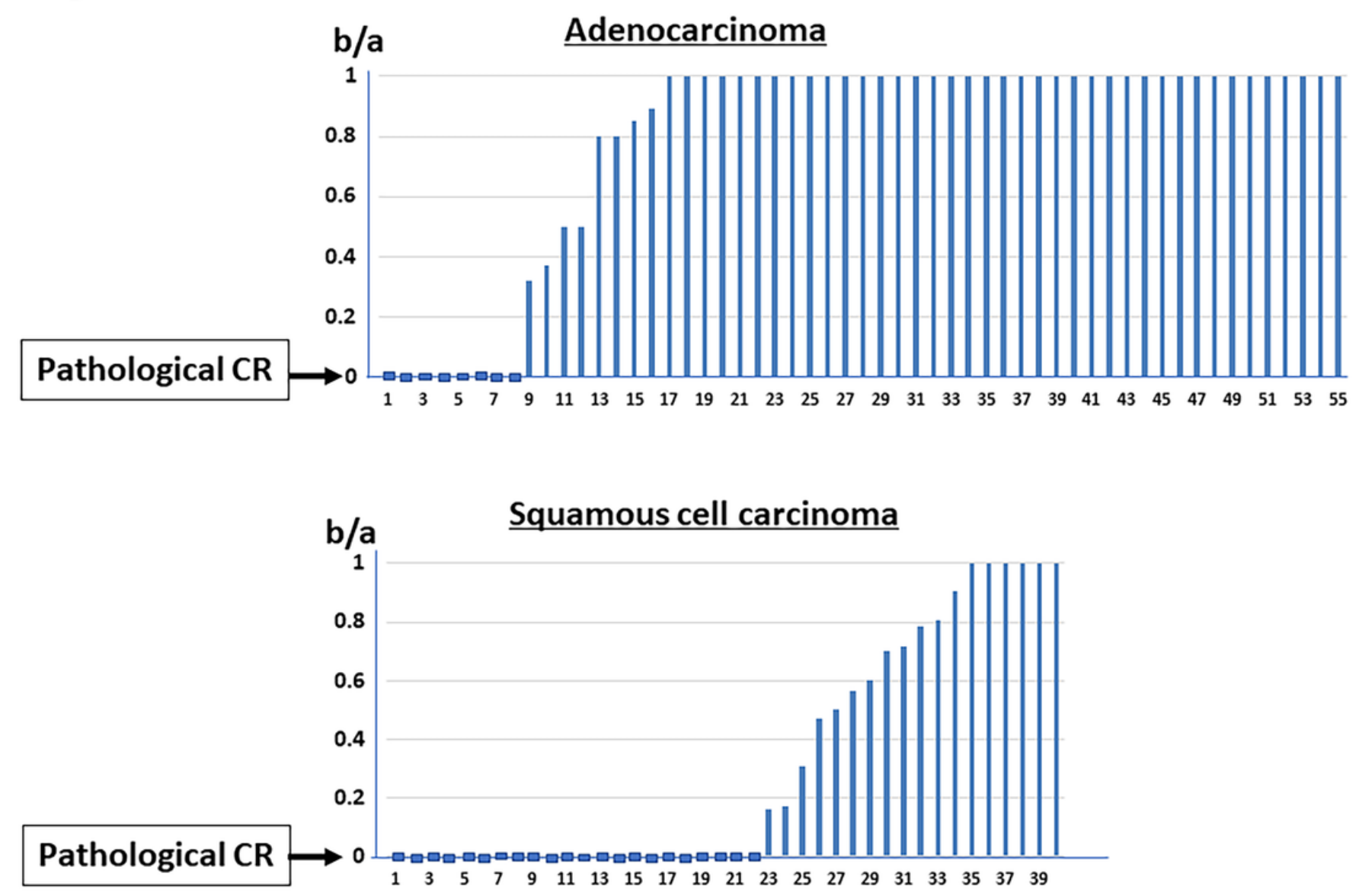

Figure 5

Waterfall plots depicting the location of the external margin of the residual tumor (b/a). Pathological CR: pathological complete remission. Of the 55 adenocarcinomas, 39 (71\%) showed the residual tumors at the periphery of the primary tumor ( $b / a=1.0)$, in contrast to only 6 of 40 squamous cell carcinomas $(15 \%)$; the difference was significant $(p<0.001)$. 


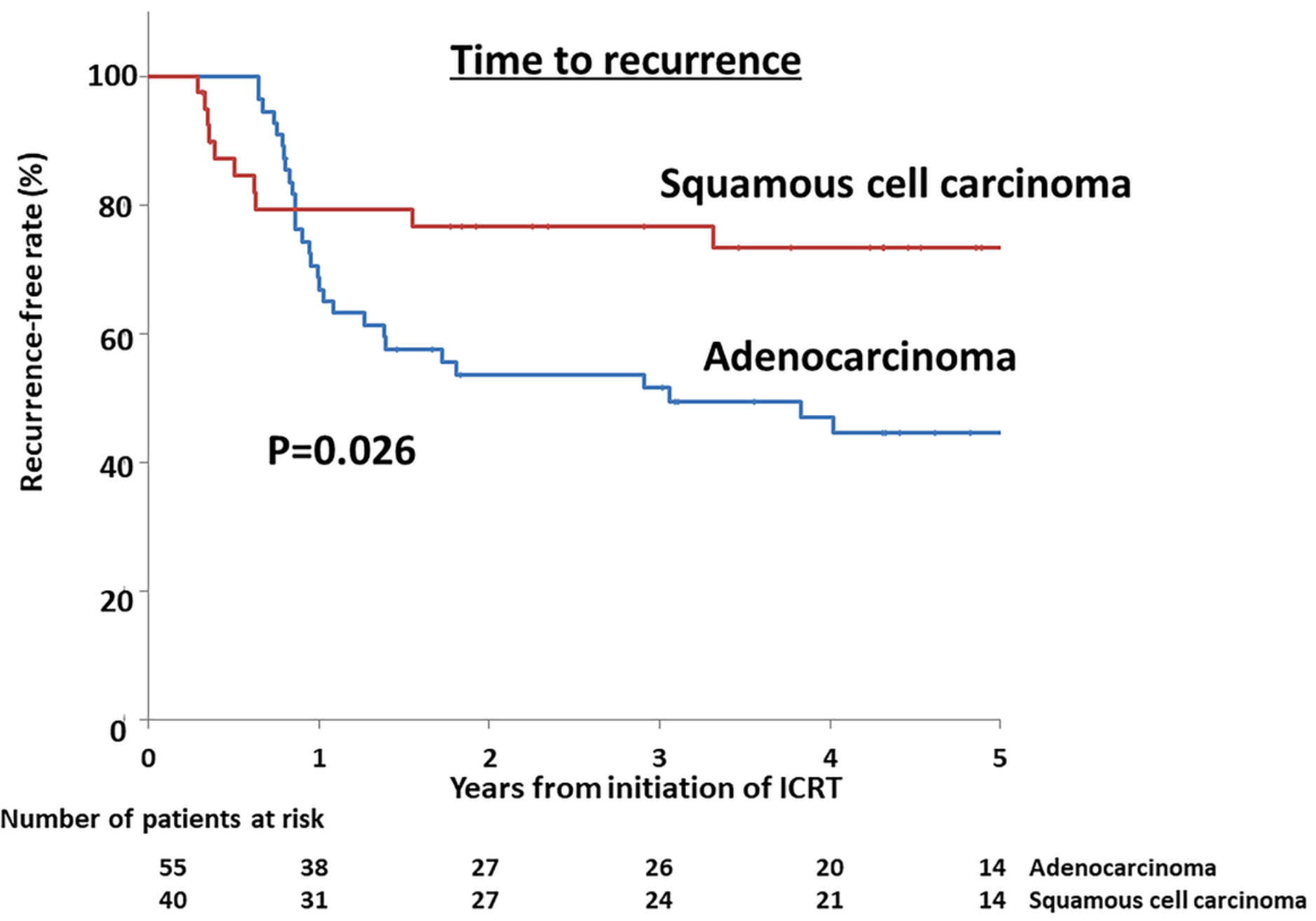

Figure 6

Time to recurrence of patients with adenocarcinoma and squamous cell carcinoma. Time to recurrence was significantly longer in patients with squamous cell carcinoma than in those with adenocarcinoma $(p=0.026)$. 


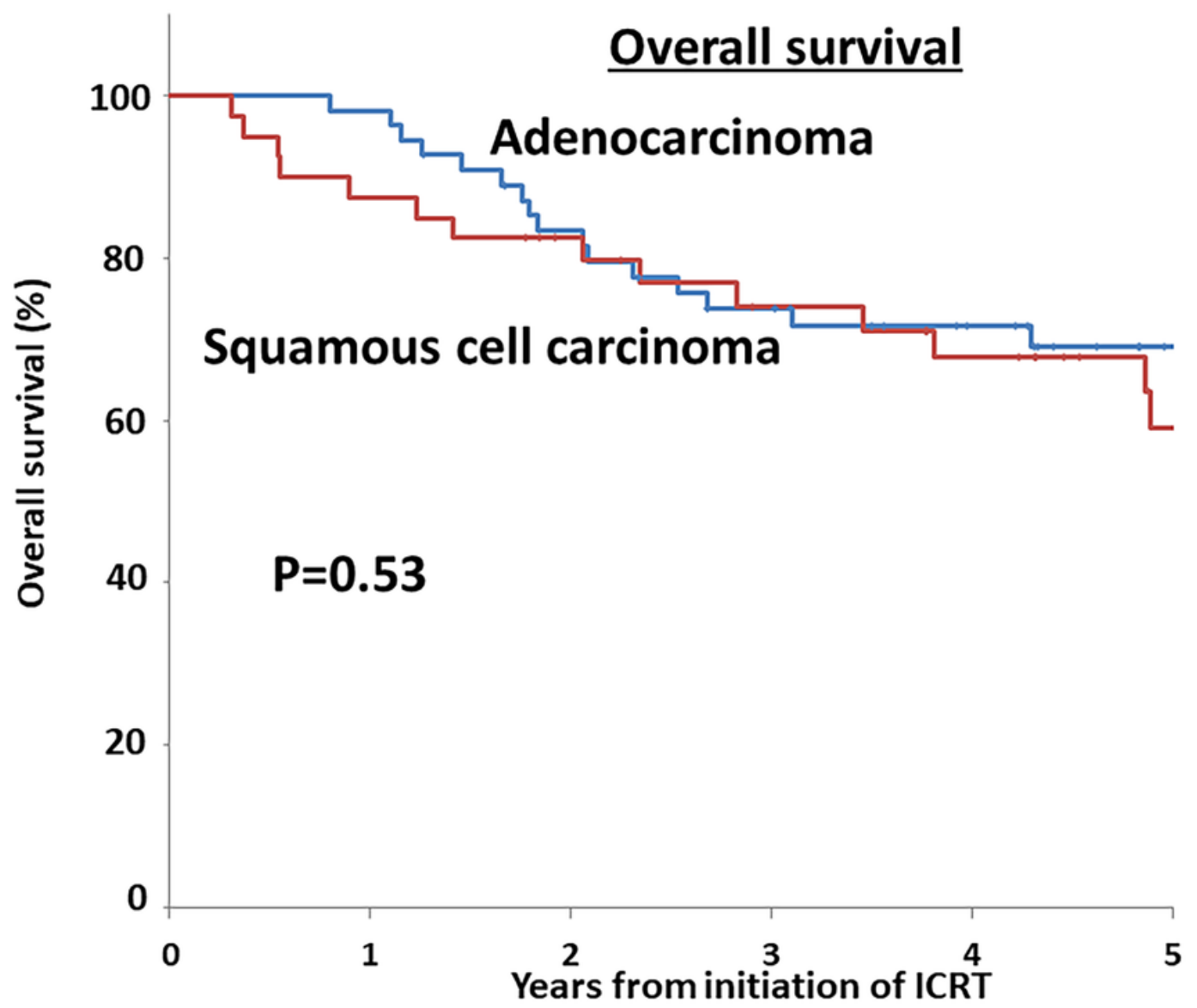

Number of patients at risk

$\begin{array}{lllllll}55 & 55 & 45 & 39 & 31 & 21 & \text { Adenocarcinoma } \\ 40 & 36 & 31 & 26 & 22 & 14 \text { Squamous cell carcinoma }\end{array}$

Figure 7

Overall survival of patients with adenocarcinoma and squamous cell carcinoma. The overall survival was not significantly different between the two $(p=0.53)$.

\section{Supplementary Files}

This is a list of supplementary files associated with this preprint. Click to download.

- Fig.E2.TIF

- Fig.E1.TIF

- 2020.5.24.TableE5.docx

- 2020.5.24.TableE4.docx

- 2020.5.24.TableE3.docx

- 2020.5.24.TableE2.docx 
- 2020.5.24.TableE1.docx

Page 19/19 